\title{
Travelling-wave solutions for some fractional partial differential equation by means of generalized trigonometry functions
}

\author{
Zakia Hammouch and Toufik Mekkaoui \\ Département de Mathématiques FST Errachidia BP 509, Boutalamine 52000 \\ Université Moulay Ismail Morocco \\ hammouch.zakia@gmail.com \\ Département de Mathématiques FST Errachidia BP 509, Boutalamine 52000 \\ Université Moulay Ismail Morocco \\ toufik_mekkaoui@yahoo.fr
}

\begin{abstract}
Making use of a Fan's sub-equation method and the Mittag-Leffler function with aid of the symbolic computation system Maple we construct a family of traveling wave solutions of the Burgers and the KdV equations. The obtained results include periodic, rational and solitonlike solutions.
\end{abstract}

Keywords: Euler function, Mittag-Leffler function, TW-solution, Generalized trigonometry functions, Fractional derivative.

\section{Introduction}

Recently it was found that many physical, chemical, biological and medical processes are governed by fractional differential equations (FDEs). As mathematical models of the phenomena, the investigation of exact solutions of (FDEs) will help one to understand these phenomena more precisely. On the other hand the investigation of the exact travelling wave solutions for nonlinear partial differential equations plays a crucial role in the study of nonlinear physical phenomena. Such solutions when they exist can help us to understand the complicated physical phenomena modeled by these equations [2]. Many powerful methods for obtaining exact solutions of nonlinear partial differential 
equations have been presented such as, Homotopy method [12], Hirota's bilinear method [7], Backlund transformation, homogeneous balance method [15], projective Riccati method [13], the sine-cosine method [14] etc.

In the present paper we aim to find exact solitary wave solutions of some nonlinear fractional partial differential equation with modified Riemann-LiouvilleJumarie derivatives. Using a sub-equation method [17] we obtain three types of solutions for the studied equations namely; two generalized hyperbolic function solutions, two generalized trigonometric function solutions and one rational solution.

\section{Preliminaries}

There are different definitions for fractional derivatives for more details see [11] and the references therein. In this paper we use the modified RiemannLiouville derivative defined by Jumarie [8]

$$
D_{x}^{\alpha} f(x)= \begin{cases}\frac{1}{\Gamma(1-\alpha)} \int_{0}^{x}(x-s)^{-\alpha-1}(f(s)-f(0)) d s, & \text { for } \quad \alpha<0, \\ \frac{1}{\Gamma(1-\alpha)} \frac{d}{d x} \int_{0}^{x}(x-s)^{-\alpha}(f(s)-f(0)) d s, & \text { for } \quad 0<\alpha<1, \\ {\left[f^{(\alpha-n)}(x)\right]^{(n)}, \quad \text { for } \quad n \leq \alpha<n+1, \quad n \geq 1 .} & \end{cases}
$$

In the following we shall outline the main idea of the fractional-Riccati equation method. For a given nonlinear fractional equation say

$$
F\left(u, u_{x}, u_{t}, D_{x}^{\alpha} u, D_{t}^{\alpha} u, \ldots\right)=0, \quad 0<\alpha \leq 1,
$$

where $D_{x}^{\alpha} u$ and $D_{t}^{\alpha} u$ are modified Riemann-Liouville derivatives of $u$ with respect to $x$ and $t$ respectively.

To determine the solution $u=u(x, t)$ explicitly, we first introduce the following transformations

$$
u=U(\xi) \quad \text { with } \quad \xi=k x+c t .
$$

which yields

$$
F\left(U, U^{\prime}, U^{\prime \prime}, D_{\xi}^{\alpha} U, D_{\xi}^{2 \alpha} U, \ldots\right)=0,
$$

Next we introduce a new variable $\varphi=\varphi(\xi)$ which is a solution of the fractional Riccati equation

$$
D_{\xi}^{\alpha} \varphi=\sigma+\varphi^{2}, \quad 0<\alpha \leq 1 .
$$


Then we propose the following series expansion as a solution of (1)

$$
u(x, t)=U(\xi)=\sum_{i=0}^{n} a_{i} \varphi^{i},
$$

where the positive integer $n$ can be found via the balancing of the highest derivative term with the nonlinear term in equation (3). In a recent paper by Zhang et al.[17] a set of five different solutions to equation (4) was introduced as follows

$$
\left\{\begin{array}{l}
-\sqrt{-\sigma} \tanh _{\alpha}(\sqrt{-\sigma} \xi), \quad \sigma<0 \\
-\sqrt{-\sigma} \operatorname{coth}_{\alpha}(\sqrt{-\sigma} \xi), \quad \sigma<0 \\
\sqrt{\sigma} \tan _{\alpha}(\sqrt{\sigma} \xi), \quad \sigma>0 \\
-\sqrt{\sigma} \cot _{\alpha}(\sqrt{\sigma} \xi), \quad \sigma>0 . \\
-\frac{\Gamma(\alpha+1)}{\xi^{\alpha}+\omega}, \quad \omega=\text { const. } \quad \sigma=0
\end{array}\right.
$$

where the generalized hyperbolic and trigonometric functions [6] are expressed by the following

$$
\begin{aligned}
& \tanh _{\alpha}(x)=\frac{E_{\alpha}\left(x^{\alpha}\right)-E_{\alpha}\left(-x^{\alpha}\right)}{E_{\alpha}\left(x^{\alpha}\right)+E_{\alpha}\left(-x^{\alpha}\right)}, \\
& \operatorname{coth}_{\alpha}(x)=\frac{E_{\alpha}\left(x^{\alpha}\right)+E_{\alpha}\left(-x^{\alpha}\right)}{E_{\alpha}\left(x^{\alpha}\right)-E_{\alpha}\left(-x^{\alpha}\right)}, \\
& \tan _{\alpha}(x)=\frac{E_{\alpha}\left(i x^{\alpha}\right)-E_{\alpha}\left(-i x^{\alpha}\right)}{i\left(E_{\alpha}\left(i x^{\alpha}\right)+E_{\alpha}\left(-i x^{\alpha}\right)\right)}, \\
& \cot _{\alpha}(x)=\frac{i\left(E_{\alpha}\left(i x^{\alpha}\right)+E_{\alpha}\left(-i x^{\alpha}\right)\right)}{E_{\alpha}\left(i x^{\alpha}\right)+E_{\alpha}\left(-i x^{\alpha}\right)},
\end{aligned}
$$

where $E_{\alpha}$ denotes the Mittag-Leffler function, given as

$$
E_{\alpha}(z)=\sum_{k=0}^{\infty} \frac{z^{k}}{\Gamma(\alpha k+1)} .
$$

\section{Applications}

In this section we give two illustrative examples, namely Burgers and the $\mathrm{KdV}$ equations. 


\subsection{Example.1}

The Burgers equation [3] is considered as one of the fundamental model equations in fluid mechanics. The equation demonstrates the coupling between diffusion and convection processes. Consider the following Burgers equation with fractional-order derivatives

$$
D_{t}^{\alpha} u+u D_{x}^{\alpha} u-\nu D_{x}^{2 \alpha} u=0 .
$$

where $\nu$ is a constant that defines the kinematic viscosity. If the viscosity $\nu=0$, the equation is called inviscid Burgers equation [16]. Our main task is to seek travelling wave solutions to equation (7). Using the wave transformation (2), we reduce equation (7) to an $\mathrm{ODE}$

$$
c^{\alpha} D_{\xi}^{\alpha} U+k^{\alpha} U D_{\xi}^{\alpha} U-\nu k^{2 \alpha} D_{\xi}^{2 \alpha} U=0 .
$$

By (4) and balancing the terms $D^{2 \alpha} u$ with the term $u D^{\alpha} u$ in (8), we obtain $n=1$. Substituting equation (5) in (8) with $n=1$ and collecting all coefficients of $\varphi^{i}, i=0, \ldots, 3$ we get the following system of algebraic equations for $a_{0}$ and $a_{1}$

$$
\left\{\begin{array}{l}
k^{\alpha} a_{1}^{2}-2 \nu k^{2 \alpha} a_{1}=0, \\
c^{\alpha} a_{1}+k^{\alpha} a_{0} a_{1}=0, \\
k^{\alpha} a_{1}^{2} \sigma-2 \nu k^{2 \alpha} a_{1} \sigma=0, \\
c^{\alpha} a_{1} \sigma+k^{\alpha} a_{1} a_{0} \sigma=0,
\end{array}\right.
$$

we find that

$$
a_{0}=-\frac{c^{\alpha}}{k^{\alpha}} \quad a_{1}=2 \nu k^{\alpha} .
$$

Accordingly we obtain

- Two hyperbolic function solutions:

$$
\begin{array}{ll}
u(x, t)=-\frac{c^{\alpha}}{k^{\alpha}}-2 \nu k^{\alpha} \sqrt{-\sigma} \tanh _{\alpha}(\sqrt{-\sigma}(k x+c t)) & \sigma<0, \\
u(x, t)=-\frac{c^{\alpha}}{k^{\alpha}}-2 \nu k^{\alpha} \sqrt{-\sigma} \operatorname{coth}_{\alpha}(\sqrt{-\sigma}(k x+c t)) & \sigma<0,
\end{array}
$$

- Two trigonometric function solutions:

$$
\begin{array}{ll}
u(x, t)=-\frac{c^{\alpha}}{k^{\alpha}}+2 \nu k^{\alpha} \sqrt{\sigma} \tan _{\alpha}(\sqrt{\sigma}(k x+c t)) & \sigma>0, \\
u(x, t)=-\frac{c^{\alpha}}{k^{\alpha}}-2 \nu k^{\alpha} \sqrt{\sigma} \cot _{\alpha}(\sqrt{\sigma}(k x+c t)) & \sigma>0,
\end{array}
$$


- One rational solution:

$$
u(x, t)=-\frac{c^{\alpha}}{k^{\alpha}}-2 \nu k^{\alpha} \frac{\Gamma(1+\alpha)}{(k x+c t)^{\alpha}+\omega} \quad \sigma>0 \quad \text { and } \quad \omega=\text { const } .
$$

\section{$3.2 \quad$ Example.2}

The Korteweg-deVries (KdV) equation arises in the study of shallow water waves [16]. In particular, the $\mathrm{KdV}$ equation is used to describe long waves traveling in canals. It is formally proved that this equation has solitary waves as solutions, hence it can have any number of solitons [5][16]. Consider the following fractional $\mathrm{KdV}$ equation with fractional-order derivatives

$$
D_{t}^{\alpha} u+6 u D_{x}^{\alpha} u+D_{x}^{3 \alpha} u=0
$$

Similarly, we use the wave transformation (2), we reduce equation (14) to an ODE

$$
c^{\alpha} D_{\xi}^{\alpha} U+6 k^{\alpha} U D_{\xi}^{\alpha} U+k^{3 \alpha} D_{\xi}^{3 \alpha} U=0,
$$

Thanks to (4) and the balancing of the terms $D_{\xi}^{3 \alpha} U$ with the term $U D_{\xi}^{\alpha} U$ in (15), we obtain $n=2$. By substituting equation(5) in (15) with $n=2$ and collecting all coefficients of $\varphi^{i}, i=0, \ldots, 5$ we get the following system of algebraic equations for $a_{0}, a_{1}$, and $a_{2}$,

$$
\left\{\begin{array}{l}
12 k^{\alpha} a_{2}^{2}+24 k^{3 \alpha} a_{2}=0 \\
18 k^{\alpha} a_{1} a_{2}+6 k^{3 \alpha} a_{1}=0 \\
2 a_{2} c^{\alpha}+12 k^{\alpha} a_{0} a_{2}+12 k^{\alpha} a_{2}^{2} \sigma+6 k^{\alpha} a_{1}^{2}+40 k^{3 \alpha} a_{2} \sigma=0 \\
8 k^{3 \alpha} a_{1} \sigma+6 k^{\alpha} a_{0} a_{1}+18 k^{\alpha} a_{1} a_{2} \sigma+c^{\alpha} a_{1}=0, \\
2 c^{\alpha} a_{2} \sigma+16 k^{3 \alpha} a_{2} \sigma^{2}+12 k^{\alpha} a_{0} a_{2} \sigma+6 k^{\alpha} a_{1}^{2} \sigma=0, \\
c^{\alpha} a_{1} \sigma+2 k^{3 \alpha} a_{1} \sigma^{2}+6 k^{\alpha} a_{0} a_{1} \sigma=0 .
\end{array}\right.
$$

From the output of Maple, we get a solution namely

$$
a_{0}=-\frac{8 k^{3 \alpha} \sigma+c^{\alpha}}{6 k^{\alpha}} \quad a_{1}=0, \quad a_{2}=-2 k^{2 \alpha} .
$$

In view of this we obtain 
- Two hyperbolic functions solution:

$$
\begin{array}{ll}
u(x, t)=-\frac{8 k^{3 \alpha} \sigma+c^{\alpha}}{6 k^{\alpha}}+2 k^{2 \alpha} \sigma \tanh _{\alpha}^{2}(\sqrt{-\sigma}(k x+c t)) & \sigma<0, \\
u(x, t)=-\frac{8 k^{3 \alpha} \sigma+c^{\alpha}}{6 k^{\alpha}}+2 k^{2 \alpha} \sigma \operatorname{coth}_{\alpha}^{2}(\sqrt{-\sigma}(k x+c t)) & \sigma<0,
\end{array}
$$

- Two trigonometric function solutions:

$$
\begin{array}{ll}
u(x, t)=-\frac{8 k^{3 \alpha} \sigma+c^{\alpha}}{6 k^{\alpha}}-2 k^{2 \alpha} \sigma \tan _{\alpha}^{2}(\sqrt{\sigma}(k x+c t)) & \sigma>0, \\
u(x, t)=-\frac{8 k^{3 \alpha} \sigma+c^{\alpha}}{6 k^{\alpha}}-2 k^{2 \alpha} \sigma \cot _{\alpha}^{2}(\sqrt{\sigma}(k x+c t)) & \sigma>0,
\end{array}
$$

- One rational solution:

$$
u(x, t)=-\frac{c^{\alpha}}{6 k^{\alpha}}-\frac{2 k^{2 \alpha} \Gamma^{2}(\alpha+1)}{\left((k x+c t)^{\alpha}+\omega\right)^{2}} \quad \omega=\text { const. } \quad \sigma=0 .
$$

\section{Conclusion}

In this paper we give some exact analytical solutions including the generalized hyperbolic function solutions, generalized trigonometric function solutions and rational solutions by using a sub-equation method. It can be concluded that the improved sub-equation method can be used for solving other nonlinear fractional partial differential equations with nonlinear terms of any order. All solutions obtained in this paper have been checked by Maple software.

\section{References}

[1] G. Adomian, Kluwer Academic Publishers, Boston, MA 1994.

[2] N.Benhamidouche, Exact solutions to some nonlinear PDEs, travelling profiles method Electronic Journal of Qualitative Theory of Differential Equations 2008, No. 15, 1-7.

[3] J.M. Burgers, Adv. Appl. Mech. 1, 171-199, (1948).

[4] E.G.Fan, Phys. Lett. A 300 (2002) 243. 
[5] W.Hereman and A. Nuseir, Math. Comput. Simulation, 43, 1327, (1997).

[6] http://mathworld.wolfram.com/GeneralizedHyperbolicFunctions.html

[7] R.Hirota, Phys. Rev. Lett. 27 (1971) 1192.

[8] G.Jumarie, Comput. Math. Appl. 51 (2006) 1367.

[9] R.M. Miura, SIAM Rev., 18, 412459, (1976).

[10] M.R.Miurs, Backlund Transformation, Springer, Berlin, 1978.

[11] K.B. Oldham and J. Spanier, The Fractional Calculus, Academic Press, New York 1974.

[12] Foad Saadi, M. Jalali Azizpour and S.A. Zahedi, Analytical Solutions of Kortweg-de Vries (KdV) Equation. World Academy of Science, Engineering and Technology 692010 171-175.

[13] Alvaro H. Salas, Exact Solutions to Kaup-Kupershmidt Equation by Projective Riccati Equations Method, Int.Math Forum Vol. 7, 2012, no. 8, $391-396$.

[14] Filiz Tascan and Ahmet Bekir, Exact Solutions of Distinct Physical Structures to Nonlinear Evolution Equations, J. Adv Research In diff Equations, Volume 2, Issue 3, 2010 pp. 1-9.

[15] M.L.Wang, Phys. Lett. A 213 (1996) 279.

[16] A.M.Wazwaz, Partial differential equations and solitary waves theory,

[17] S.Zhang,H-Q.Zhang, Physics Letters A 375 (2011) 1069-1073 